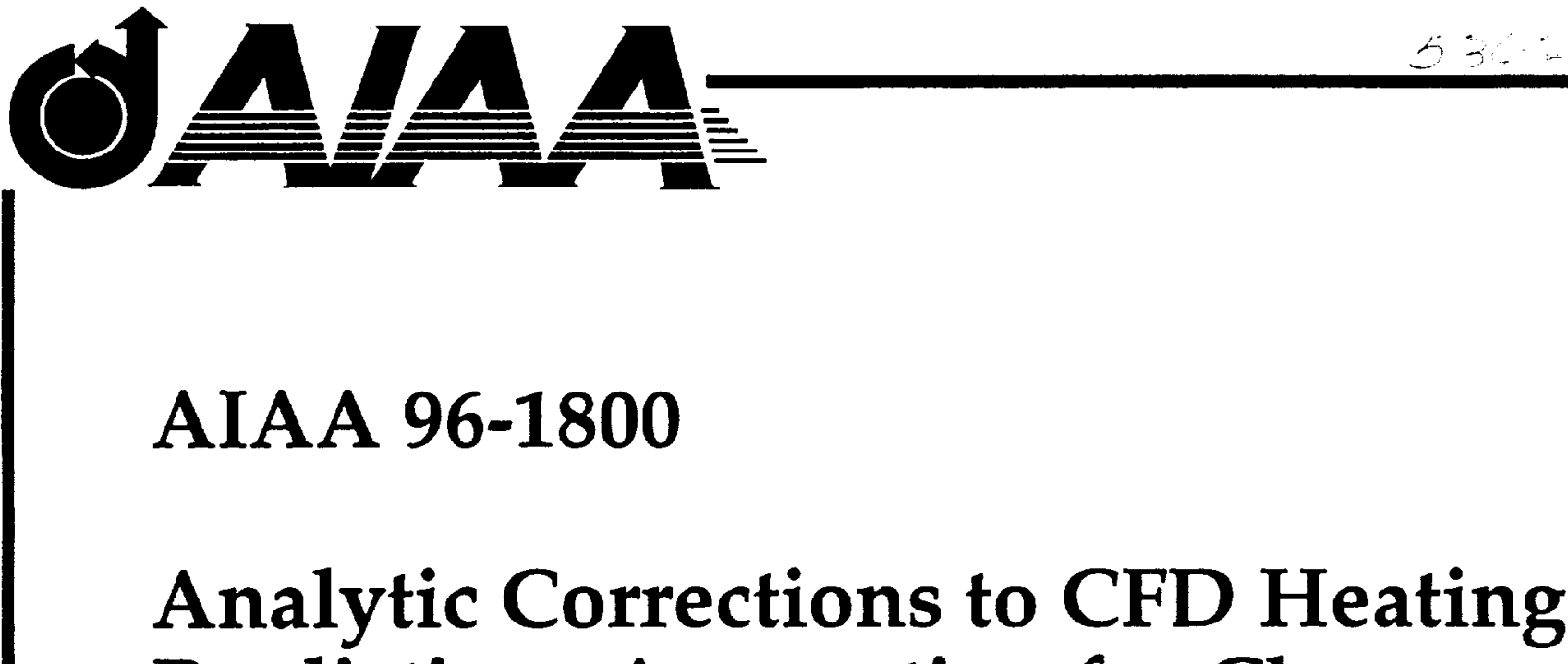 Predictions Accounting for Changes in Surface Catalysis
}

Peter A. Gnoffo

NASA Langley Research Center

Hampton, VA 23681-0001

George R. Inger

Iowa State University

Ames, IA 50011-3231

\section{1st AIAA Thermophysics Conference June 17-20, 1996/New Orleans, LA}





\title{
Analytic Corrections to CFD Heating Predictions Accounting for Changes in Surface Catalysis
}

\author{
Peter A. Gnoffo * \\ NASA Langley Research Center \\ Hampton, Virginia 23681-0001 \\ and \\ George R. Inger ${ }^{\dagger}$ \\ Iowa State University \\ Ames, Iowa 50011-3231
}

\begin{abstract}
$\underline{\text { Abstract }}$
Integral boundary-layer solution techniques applicable to the problem of determining aerodynamic heating rates of hypersonic vehicles in the vicinity of stagnation points and windward centerlines are briefly summarized. A new approach for combining the insight afforded by integral boundary-layer analysis with comprehensive (but time intensive) computational fluid dynamic (CFD) flowfield solutions of the thin-layer Navier-Stokes equations is described. The approach extracts CFD derived quantities at the wall and at the boundary layer edge for inclusion in a post-processing boundary-layer analysis. It allows a designer at a workstation to address two questions, given a single CFD solution. (1) How much does the heating change for a thermal protection system with different catalytic properties than was used in the original CFD solution? (2) How does the heating change at the interface of two different TPS materials with an abrupt change in catalytic efficiency? The answer to the second question is particularly important, because abrupt changes from low to high catalytic efficiency can lead to localized increase in heating which exceeds the usually conservative estimate provided by a fully catalytic wall assumption.
\end{abstract}

*Aerospace Engineer, Aerothermodynamics Branch, Gas Dynamics Division, Associate Fellow AIAA.

${ }^{\dagger}$ Professor, Dept. of Aerospace Engr. \& Engr. Mechanics, Associate Felow AIAA

Copyright (C) 1996 by the American Institute of Aeronautics and Astronautics, Inc. No copyright is asserted in the United States under Title 17, U.S. Code. The U.S Government has a royalty-free license to exercise all rights under the copyright claimed herein for Governmental purposes. All other rights are reserved by the copyright owner.

\section{Nomenclature}

$C \quad$ relaxation coefficient in Method 2

$C_{D} \quad$ equilibrium constant, $p_{e} e^{\theta_{D}}\left[\frac{\alpha_{e}^{2}}{1 \cdot-\alpha_{e}^{2}}\right]_{E Q}$

$\hat{C}_{p} \quad$ specific heat of molecules, $\mathrm{J} / \mathrm{kmole}-\mathrm{K}$

$h \quad$ enthalpy per unit mass of mixture, $\mathrm{J} / \mathrm{kg}$

$h_{D}$ heat of formation per unit mass of species, $\mathrm{J} / \mathrm{kg}$

$I_{(1-3, D)}$ reaction integrals

$k_{r} \quad$ recombination rate coefficient, $\mathrm{m}^{6} / \mathrm{kmole}^{2}$-s

Le Lewis number

$p \quad$ pressure, $\mathrm{N} / \mathrm{m}^{2}$

Pr Prandtl number

$q$ surface heating, $W / \mathrm{m}^{2}$

$Q$ heat transfer function

$r \quad$ ratio of velocity gradients

$R \quad$ radius of curvature, $\mathrm{m}$

$R_{s} \quad$ gas constant for species $s \mathrm{~J} / \mathrm{kmole}-\mathrm{K}$

$R_{u} \quad$ univ. gas constant, $8314.3 \mathrm{~J} / \mathrm{kmole}-\mathrm{K}$

Sc Schmidt number

$T$ temperature, $\mathrm{K}$

$T_{D} \quad$ characteristic temperature of dissociation, $\mathrm{K}$

$u, w \quad$ streamwise and crossflow velocites, $\mathrm{m} / \mathrm{s}$

$V$ total velocity, $\mathrm{m} / \mathrm{s}$

$x, z \quad$ streamwise and crossflow distances, $\mathrm{m}$

$Z$ bridging function

$\alpha \quad$ mass fraction of atoms

$\beta \quad$ velocity gradient, $1 / \mathrm{s}$

$\gamma \quad$ catalytic efficiency of surface

$\Gamma^{*} \quad$ composite Dahmkohler number

$\Gamma_{c}, \tilde{\Gamma}_{c}$ Dahmkohler numbers for surface catalysis

$\Gamma_{D} \quad$ Dahmkohler number for dissociation dominated flow

$\Gamma_{G} \quad$ Dahmkohler number for recombination dominated flow

$\rho$ density, $\mathrm{kg} / \mathrm{m}^{3}$

$\mu \quad$ viscosity, $\mathrm{N}-\mathrm{s} / \mathrm{m}^{2}$

$\theta \quad$ temperature ratio $T / T_{e}$

$\theta_{D} \quad$ temperature ratio $T_{D} / T_{e}$

$\xi, \eta \quad$ Levy-Lees transformation coordinates

$\chi \quad$ parameter $u_{e}(d \xi / d x)(1+r) / \xi, 1 / \mathrm{s}$ 
Superscripts

$+\quad$ value immediately following jump

- $\quad$ value immediately preceding jump

Subscripts

$\infty \quad$ free stream

$0 \quad$ computed using $\gamma=0$

1 computed using $\gamma=1$

$e$ boundary-layer edge

$E Q$ equilibrium

$F \quad$ frozen

$i, j \quad$ CFD mesh point location

$J$ location of jump in surface catalysis

$N$ nitrogen atom

$O \quad$ oxygen atom

$w \quad$ wall

$\gamma \quad$ computed using $\gamma=\gamma(T)$

$\gamma \rightarrow 1$ computed across discontinuity in $\gamma$ from $\gamma=\gamma(T)$ to $\gamma=1$

\section{Introduction}

Integral boundary-layer solution techniques applicable to the problem of determining aerodynamic heating rates of hypersonic vehicles in the vicinity of stagnation points, windward centerlines, and sweptwing leading edges are discussed in the literature. ${ }^{1-4}$ The analyses include effects of finite-rate gas chemistry across the boundary layer and finite-rate catalysis of atom recombination at the surface. A new approach for combining the insight afforded by integral boundarylayer analysis with comprehensive (but time intensive) computational fluid dynamic (CFD) flowfield solutions of the thin-layer Navier-Stokes equations is described. The approach extracts CFD derived quantities at the wall and at the boundary layer edge for inclusion in a post-processing boundary-layer analysis. The postprocessed data base allows a designer at a workstation to address two questions, given a single CFD solution. (1) How much does the heating change for a thermal protection system with different catalytic properties then was used in the original CFD solution? (2) How does the heating change at the interface of two different TPS materials with an abrupt change in catalytic efficiency? The answer to the second question is particularly important, because abrupt changes from low to high catalytic efficiency can lead to localized increase in heating which exceeds the usually conservative estimate provided by a fully catalytic wall assumption. For a given trajectory point, the approach uses a single
CFD solution obtained with a known variation of catalytic efficiency over the vehicle surface. Changes to CFD baseline heating levels are calculated as a function of changes in catalytic efficiency derived from integral boundary-layer solution techniques that utilize CFD generated edge and wall conditions.

The present paper focuses on flow over a sphere at several trajectory points characteristic of reentry of a representative winged Single Stage to Orbit (SSTO) vehicle. CFD calculations are made for three wall catalysis models at each of these points. The models include noncatalytic, finite catalytic (Shuttle tile), and fully catalytic wall boundary conditions. The post processed integral boundary-layer corrections using each of three baseline CFD heating results are compared to actual CFD calculations for the corresponding offbaseline case.

Also presented are the results of preliminary tests on vehicles of realistic geometric complexity appropriate for Access-to-Space studies. Relative differences in predicted heating levels between single CFD runs with post-processed corrections and multiple CFD runs using different wall catalysis models are discussed.

The goal of this research is to create a stand-alone post-processing tool which can be used in preliminary design of thermal protection systems (TPS) for hypersonic vehicles. The tool would make extensive use of a small number of CFD solutions computed using a relatively simple surface catalysis model. Design iterations are conducted without need of additional CFD runs until convergence on a single concept is achieved, at which point CFD could be used to provide a final check and/or recalibration point.

\section{Baseline CFD Solution Algorithm}

The computational fluid dynamic (CFD) baseline solutions for real gas, viscous analysis are provided by the Langley Aerothermodynamic Upwind Relaxation Algorithm (LAURA) ${ }^{5-7}$ Comparisons to experimental data for hypersonic flows in air are documented in the literature ${ }^{8-12}$ The code employs upwind-biased, point-implicit relaxation. Inviscid fluxes are approximated with Roe's averaging, ${ }^{13}$ eigenvalue limiting (similar to Harten ${ }^{14}$ ), and Yee's symmetric total variation diminishing scheme. ${ }^{15}$ Viscous fluxes are approximated with central differences. A model for surface catalytic efficiency ${ }^{16} \gamma(T)$ used in the present work is defined by

$$
\begin{aligned}
& \gamma_{O}=40 . e^{-11440 . / T_{w}} \quad 1435<T_{w}<1580 \\
& \gamma_{O}=39.910^{-9} e^{21410 . / T_{w}} \quad 1580<T_{w}<1845 \\
& \gamma_{N}=0.061 e^{-2480 . / T_{w}} \quad 1410<T_{w}<1640 \\
& \gamma_{N}=0.00061 e^{5090 . / T_{w}} \quad 1640<T_{w}<1905
\end{aligned}
$$


The catalytic recombination rate $K_{w, s}$ for species $s$ is then defined by

$$
K_{w, s}=\gamma_{s}\left(T_{w}\right) \sqrt{R_{s} T_{w} / 2 \pi}
$$

\section{Integral Boundary-Layer Method}

\section{Highlights}

Integral boundary-layer theory for evaluating nonequilibrium effects on surface heating has been described previously..$^{2-4}$ Key features of the analysis are reviewed here. First, the model accounts for both finite catalysis of the surface and finite reactivity of the boundary layer. The finite catalysis of the surface enters the analysis through the Dahmkohler number, $\Gamma_{c}$, the ratio of atom recombination time at the surface and a characteristic local diffusion time.

$$
\Gamma_{c}=\left[\frac{2 \rho_{w}}{\mu_{w} \chi}\right]^{1 / 2} S c K_{w}
$$

The finite reactivity of the boundary layer enters the analysis through the Dahmkohler number, $\Gamma_{G}$, and $\Gamma_{D}$ the ratio of characteristic local flow time to gas phase reaction time. In the case of recombination dominated boundary layers (near stagnation points)

$$
\Gamma_{G}=\frac{4 k_{r} T_{e}^{-2} p_{e}^{2}}{R_{u}^{2} \chi}
$$

In the case of dissociation dominated boundary layers (near windside centerline where viscous shear raises temperatures in the boundary layer)

$$
\Gamma_{D}=\frac{C_{D}}{p_{e}} \Gamma_{G}
$$

Second, analytic solutions to the governing boundary-layer equations can be made with simplifying approximations in the vicinity of the vehicle stagnation point (nose region), swept leading edge region, and windward centerline region. The parameter $\chi$,

$$
\chi=u_{e}(d \xi / d x)(1+r) / \xi
$$

is a function of the Levy-Lees transformed coordinate $\xi$ and takes on relatively simple, limiting values in these regions. The Levy-Lees transformed coordinate is defined by

$$
\xi=\int_{0}^{x} \rho_{w} \mu_{w} u_{e} d x
$$

Three-dimensional effects are included with the parameter $1+r$, where $r$ is the ratio of velocity gradients at the edge of the boundary layer.

$$
r=\left(d w_{e} / d z\right) /\left(d u_{e} / d x\right)
$$

Global Change in Nose Region - Method 1

The post-processing algorithm used to correct for changes in surface heating associated with global change of the surface catalysis model in the nose region of a hypersonic vehicle is denoted Method 1. Surface heating in the nose region can be expressed ${ }^{2}$ as an appropriate interpolation of an equilibrium boundarylayer heating rate $Q_{w, E Q}$ and a frozen boundary-layer heating rate $Q_{w, F}$ by

$$
Q_{w}=Q_{w, E Q}+Z\left(\Gamma^{*}\right)\left(Q_{w, F}-Q_{w, E Q}\right)
$$

In Eq. 9 the variable $Q$ is a dimensionless heating rate, related to the dimensional heating rate $q_{w}$ by

$$
Q_{w}=q_{w} \frac{-P r / \hat{C}_{p} T_{e}}{\sqrt{\rho_{w} \mu_{w} \chi}}
$$

The limiting form of Eq. 10 for the stagnation point can be expressed by

$$
Q_{w, i, j}=q_{w, i, j} \frac{-P r_{w, i, j} / \hat{C}_{p} T_{e, i, j}}{\sqrt{\rho_{w, i, j} \mu_{w, i, j} \beta_{e, i, j}\left(1+r_{i, j}\right)}}
$$

where $\beta_{e, i, j}(1+r)$ is the limiting form of $\chi$ in the stagnation region and $\beta_{e, i, j}$ is the streamwise velocity gradient which, using Newtonian theory, can be approximated by

$$
\beta_{e, i, j}=\frac{d u_{e, i, j}}{d x} \approx \frac{1}{R_{x, i, j}} \sqrt{\frac{2\left(p_{e, i, j}-p_{\infty}\right)}{\rho_{e, i, j}}}
$$

While Eqs. 11 and 12 are rigorously true only at the stagnation point, they are used throughout the nose region at every mesh point $(i, j)$ of the baseline CFD solution in subsequent development of Method 1.

The equilibrium boundary-layer heating rate $Q_{w, E Q}$ reference value at each mesh point $(i, j)$ of the nose region is defined ${ }^{2}$ by

$$
\begin{gathered}
Q_{w, E Q, i, j}=0.47 P r_{w, i, j}^{1 / 3}\left(1-\theta_{w}\right. \\
\left.\quad+\sqrt{P r_{w}} \frac{u_{c}^{2}}{2 \hat{C}_{p} T_{e}}+L e_{w}^{0.52} \frac{\alpha_{e} h_{D}}{\hat{C}_{p} T_{e}}\right)
\end{gathered}
$$

The reference value for frozen boundary-layer heating rate $Q_{w, F}$ at each mesh point $(i, j)$ of the nose region is defined ${ }^{17}$ by

$$
\begin{aligned}
& Q_{w, F, i, j}=0.47 \operatorname{Pr}_{w, i, j}^{1 / 3}\left(1-\theta_{w}\right. \\
& \left.\quad+\sqrt{\operatorname{Pr}_{w}} \frac{u_{c}^{2}}{2 \bar{C}_{p} T_{e}}+L e_{w}^{0.67} \frac{\alpha_{c} h_{p}}{\bar{C}_{p} T_{e}} \frac{\Gamma_{c}}{1+\Gamma_{c}}\right)_{i, j}
\end{aligned}
$$

For stagnation region flow, the normalized kinetic energy term $\frac{u_{e, i, j}^{2}}{2 \hat{C}_{p} T_{e, i, j}}$ is small and can be omitted from both Eq. 13 and 14 . Equation 14 accounts for finite surface catalysis through the Dahmkohler number $\tilde{\Gamma}_{c, i, j} \equiv \Gamma_{c, i, j} /\left(0.47 S c^{1 / 3}\right)$ but does not include effects of reactions across the boundary layer. 
The bridging function $Z\left(\Gamma^{*}\right)$ in Eq. 9 varies from 0 (equilibrium limit with three body recombination rates much faster than local flow rates) to 1 (frozen limit with three body recombination rates much slower than local flow rates). It is derived from a fundamentally based analysis of the atomic species conservation equations and is given by

$$
Z\left(\Gamma^{*}\right) \approx \frac{3 \sqrt{1+16 \Gamma_{i, j}^{*} / 9}-1}{2+4 \Gamma_{i, j}^{*}}
$$

where $\Gamma_{i, j}^{*}$ is a composite Dahmkohler number defined at each surface mesh point by

$$
\Gamma_{i, j}^{*}=\alpha_{e, i, j}\left[\frac{I_{1}+2 \tilde{\Gamma}_{c} I_{2}+\tilde{\Gamma}_{c}^{2} I_{3}}{\left(1+\tilde{\Gamma}_{c}\right)^{2}}\right]_{i, j} \Gamma_{G, i, j}
$$

The integrals $I_{1}, I_{2}$, and $I_{3}$ account for integrated effects of reactions across the boundary layer on heating rates. They are tabulated ${ }^{3}$ and curve fits are provided.

$$
\begin{aligned}
& I_{1} \approx 4.80\left(0.50 / S c_{w}\right)^{0.45} \theta_{w}^{0.80(1-w)-1.84} \\
& I_{2} \approx 1.80\left(0.50 / S c_{w}\right)^{0.12} \theta_{w}^{0.63(1-w)-1.15} \\
& I_{3} \approx 0.93\left(0.50 / S c_{w}\right)^{-0.22} \theta_{w}^{0.41(1-w)-0.65}
\end{aligned}
$$

These integral approximations are valid over a parameter range $0.04 \leq \theta_{w} \leq 0.50$ and $-2 \leq \omega \leq 0$. In the test problems considered here it has been assumed that recombination of atomic oxygen can be used to characterize the reaction integrals in $\mathrm{Eq} .17$; consequently, $k_{r}=5.610^{11} T^{\omega}$ with $\omega=-1.0$. Values of the bridging function in Eq. 15 were found to be substantially characteristic of frozen boundary-layer flow for all four points in Table 1.

The following approximations have been employed in Method 1 to simplify extraction of CFD derived quantities. Because energy is used as a primary variable and temperature is a derived quantity in the CFD analysis we substitute $h-\alpha h_{D}$ for $C_{p} T$ where $\alpha h_{D}=$ $\sum_{s} \alpha_{s} h_{D, s}$ and we substitute $\left(h-\alpha h_{D}\right) /\left(h-\alpha h_{D}\right)_{e}$ for $\theta$ in the above equations. In order to model the catalytic effect of the surface on homogeneous recombimation of both nitrogen and oxygen atoms in Eq. 14 we evaluate

$$
\alpha h_{D} \frac{\tilde{\Gamma}_{c}}{1+\tilde{\Gamma}_{c}} \approx \alpha_{O} h_{D, O} \frac{\tilde{\Gamma}_{c, O}}{1+\tilde{\Gamma}_{c, O}}+\alpha_{N} h_{D, N} \frac{\tilde{\Gamma}_{c, N}}{1+\tilde{\Gamma}_{c, N}}
$$

Method 1 corrections to a baseline CFD solution are made using

$$
\begin{aligned}
& q_{w}\left(\gamma_{\text {new }}(T)\right)=q_{w}(\text { CFD baseline }) \\
& \quad+q_{w}\left(\text { Method } 1, \gamma_{\text {new }}(T)\right)-q_{w}\left(\text { Method } 1, \gamma_{\text {old }}(T)\right)
\end{aligned}
$$

In the present application of Method 1, no attempt is made to account for changes to CFD extracted quantities at the wall corresponding to the new surface catalysis model.
Local, Discontinuous Change in Nose Region, Method 2

More complete details of the following analysis may be found in a companion paper. ${ }^{1}$ The postprocessing algorithm used to correct for changes in surface heating associated with an abrupt, local change of the surface catalysis model in the nose region of a hypersonic vehicle is denoted Method 2. It is assumed that a change in TPS material occurs at a juncture (jump) location $\xi_{J}$. Initially, it will also be assumed that the boundary-layer gas phase can be treated as frozen. Upstream and downstream of $\xi_{J}$, both $\alpha_{e}$ and $\Gamma_{c}$ vary rather slowly compared with $\alpha_{w}$ itself in the relaxation zone, and hence are well approximated by local constants. The theory predicts a variation in $\alpha_{w}$ under these constraints as

$$
\alpha_{w}=C \xi^{-\left(1+\tilde{\Gamma}_{c}^{+}\right) / 2}+\frac{\alpha_{e}}{1+\tilde{\Gamma}_{c}^{+}} \quad\left(\xi \geq \xi_{J}\right)
$$

Even though $\tilde{\Gamma}_{c}$ is discontinuous across the juncture the variation of $\alpha_{w}$ across the juncture must be continuous (though its gradient will be discontinuous). Consequently, the constant $C$ is proportional to the jump in $\tilde{\Gamma}_{c}$ and defined by

$$
C=\alpha_{e}\left(\frac{1}{1+\tilde{\Gamma}_{c}^{-}}-\frac{1}{1+\tilde{\Gamma}_{c}^{+}}\right) \xi_{J}^{\left(1+\tilde{\Gamma}_{c}^{+}\right) / 2}
$$

Examination of Eqs. 20 and 21 reveals two features of the relaxation process. First, the further downstream the jump is, the longer it takes to adjust to the new catalytic condition. Second, the more catalytic the surface, the shorter the relaxation distance for any imposed $\Delta \Gamma_{c}$.

The diffusional contribution to the heat transfer function $Q_{w}$ will jump discontinuously across the juncture because of the discontinuous change in the gradient $d \alpha_{w} / d \eta=\Gamma_{c} \alpha_{w}$.

$$
\begin{gathered}
Q_{w}=0.47 \operatorname{Pr}^{1 / 3}\left[1-\theta_{w}+\frac{\sqrt{\operatorname{Pr} u_{c}^{2}}}{2 \hat{C}_{p} T_{e}}+L e^{-1 / 3} \frac{\alpha_{e} h_{D}}{\hat{C}_{p} T_{e}}\right] \\
\frac{0.47 S c^{1 / 3} h_{D}}{\tilde{C}_{p} T_{e}}\left[\tilde{\Gamma}_{c}(L e-1)-1\right] \alpha_{w}
\end{gathered}
$$

The application of Eq. 22 in Method 2 to correct CFD baseline results obtained without the local change in surface catalysis follows the example set in Method 1. Note that in Eq. 22 the first term represents the continuous part of the solution across the juncture and the second part represents the jump effect associated with the diffusional term. When the change in $Q_{w}$ is computed between the baseline case and the case with a jump in surface catalytic efficiency the contribution from the continuous part exactly cancels.

Though not presented here, the assumption of frozen gas phase in the boundary layer can be eliminated. In this case, a local iteration is required to account for the sudden change in boundary condition on the reaction integral. Inclusion of this effect was not significant in the test cases which follow. 
Generalization for Complete Vehicle, Method $1 \mathrm{G}$

Equations 13-14 must retain the normalized kinetic energy terms when treating flow away from the stagnation region. Equations 11-14 in Method $1 \mathrm{em}-$ ploy the limiting form of the parameter $\chi$ at the stagnation point and utilize this result in the entire nose region. This approximation fails away from the nose region, and a better evaluation of parameter $\chi$ is required. Numerical approximations to $\chi$ based on flat plate theory for downstream locations are also unsatisfactory. The available CFD solution may be exploited by solving for $\chi$ such that the integral boundary-layer solution for $q_{w}$ matches the known CFD solution for $q_{w}$. This treatment avoids the numerical complexity of a detailed integration of Eq. 7 along streamlines. This same value of $\chi$ is then used in the integral boundarylayer formulation for the new values of catalicity (and wall temperature, if required) to compute the new heating distribution. The approximation for $\chi$ ignores integrated effects of changes in surface temperature along the surface streamline, but works well in limited tests performed to date.

In the most general case, viscous dissipation of kinetic energy tends to produce a local maximum temperature within the boundary layer away from the stagnation region. The higher temperature may lead to dissociation dominated chemistry within the boundary layer. Inclusion of this effect leads to the following correction which is added to Eq. 14:

$$
\Delta Q_{w, F}=-0.47 \operatorname{Pr}_{w}^{1 / 3} \Gamma_{D} I_{D} \frac{h_{D}}{\hat{C}_{p} T_{e}}\left[1-\frac{L e_{w} \tilde{\Gamma}_{c}}{1+\tilde{\Gamma}_{c}}\right]
$$

where $I_{D}$ is a reaction integral, discussed and tabulated by Inger. ${ }^{2}$

\section{$\underline{\text { Test Cases }}$}

Two sets of tests for hypersonic flow over a $0.6 \mathrm{~m}$ radius sphere are presented. In the first set of tests, surface heating rates are computed for three different wall catalytic boundary conditions at trajectory points defined in Table 1. The wall temperature in each case is defined by a radiative equilibrium wall boundary condition computed with $\gamma=\gamma(T)$ and emissivity $\epsilon=0.9$. Both the fully catalytic $(\gamma=1)$ and noncatalytic $(\gamma=0)$ solutions were obtained with the wall temperature distribution obtained in the finitecatalytic $(\gamma=\gamma(T))$ case. This specification was maintained to eliminate variations in wall temperature as a cause of differences between the CFD and the integral boundary-layer heating predictions. Actual variations in radiative equilibrium surface temperatures that would be expected in the general case would be handled by a local iteration to obtain self consistent heating and temperature distributions.

Table 1 - Trajectory and Heating

\begin{tabular}{|c|c|c|c|c|c|c|}
\hline $\begin{array}{c}\mathrm{Pt} \\
- \\
\end{array}$ & $\begin{array}{l}V_{\infty}, \\
\mathrm{m} / \mathrm{s}\end{array}$ & $\begin{array}{c}\rho_{\infty} \\
\mathrm{kg} / \mathrm{m}^{3}\end{array}$ & $\begin{array}{c}T_{\infty} \\
\mathrm{K}\end{array}$ & $q_{1}$ & $\begin{array}{c}q_{\gamma}, \\
W / \mathrm{cm}^{2}\end{array}$ & $q_{0}$ \\
\hline$\overline{1}$ & 5493. & $2.00^{-4}$ & 238 & 50.5 & 41.1 & 24.4 \\
\hline 2 & 4440. & $3.53^{-4}$ & 250 . & 34.3 & 30.2 & 20.2 \\
\hline 3 & 3551. & $6.64^{-4}$ & 264. & 22.2 & 18.6 & 14.6 \\
\hline 4 & 2974. & $1.01^{-3}$ & 271. & 15.5 & 14.6 & - \\
\hline
\end{tabular}

In the second set of tests, an abrupt change in surface catalysis (from finite-catalytic to fully-catalytic) is introduced on the sphere for trajectory point 1 of Table 1 at $s / R=\pi / 6$. Such a change would approximately correspond to use of a glassy coated surface at the stagnation point followed by polished (non-oxidized), metallic surface. The integral boundary-layer methodology is used to predict both the overshoot in heating rate at the juncture and the relaxation distance to the new heating level corresponding to $\gamma=1$. Wall temperature distributions are held fixed at the radiative equilibrium values corresponding to the finite-catalytic solution as described in the previous paragraph.

\section{Global Changes to Surface Catalysis}

In Fig. 1a, the non-catalytic solution is treated as the available CFD baseline solution. This baseline CFD data set has been post-processed using Method 1 to obtain corrected heating levels associated with changes in the surface catalysis model to finite catalytic and to fully catalytic. The actual CFD heating predictions with the new surface catalysis models are compared with the results of Method 1 in Fig. 1a. Comparisons are generally very good, with a maximum difference in predicted stagnation point heating level occurring for $\gamma=1$ case equal to $10 \%$.

Similar comparisons are made in Fig. $1 \mathrm{~b}$ for point 1 except that the finite-catalytic CFD solution is used as a baseline and post-processed corrections are derived for the fully-catalytic and non-catalytic surfaces. Comparisons with CFD heating predictions are within approximately $10 \%$ over the entire surface. The fullycatalytic CFD solution is used as a baseline in Fig. 1c. The finite-catalytic prediction from Method 1 is within $10 \%$ of the CFD prediction using this baseline; however, the non-catalytic prediction is approximately $30 \%$ low as compared to the CFD result. Alternative methods for extracting and averaging boundary-layer quantities to improve this comparison while maintaing good comparisons from other baselines across the entire trajectory continue to be explored. The Lewis number profile across the boundary layer is sensitive to the catalytic boundary condition and an additional correction within the theory may be advised. 


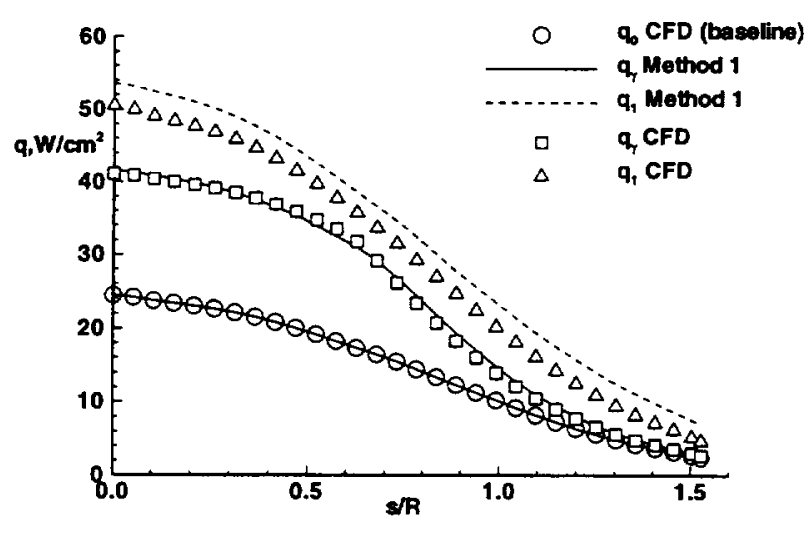

(a) CFD baseline at $\gamma=0$

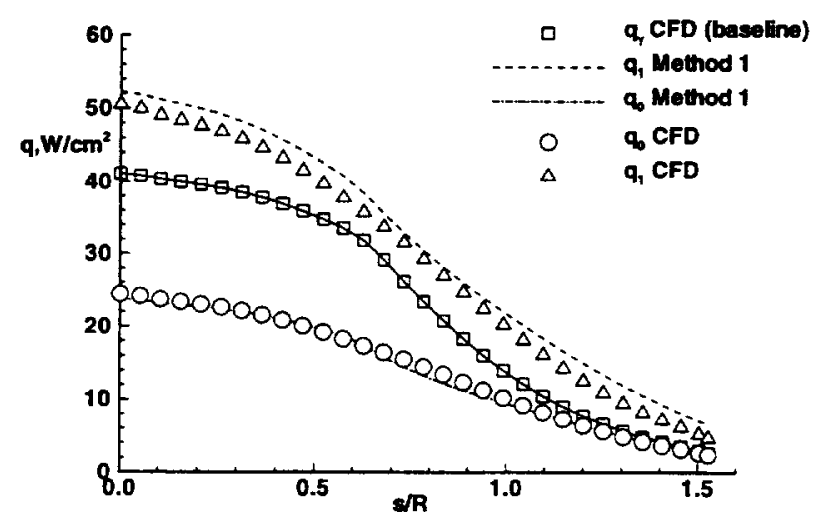

(b) CFD baseline at $\gamma=\gamma(T)$

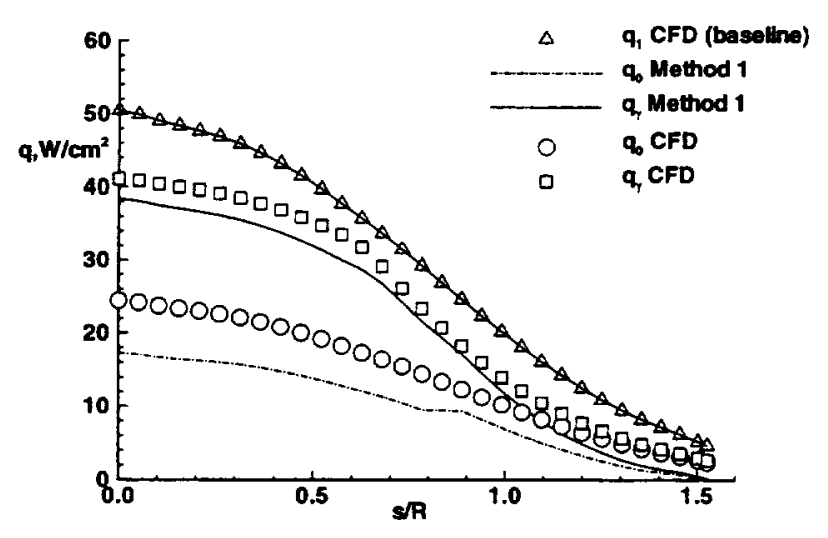

(c) CFD baseline at $\gamma=1$

Figure 1: Comparison of CFD heating levels (symbols) with analytic corrections (lines) derived from CFD baseline for point 1 as function of three surface catalysis models.

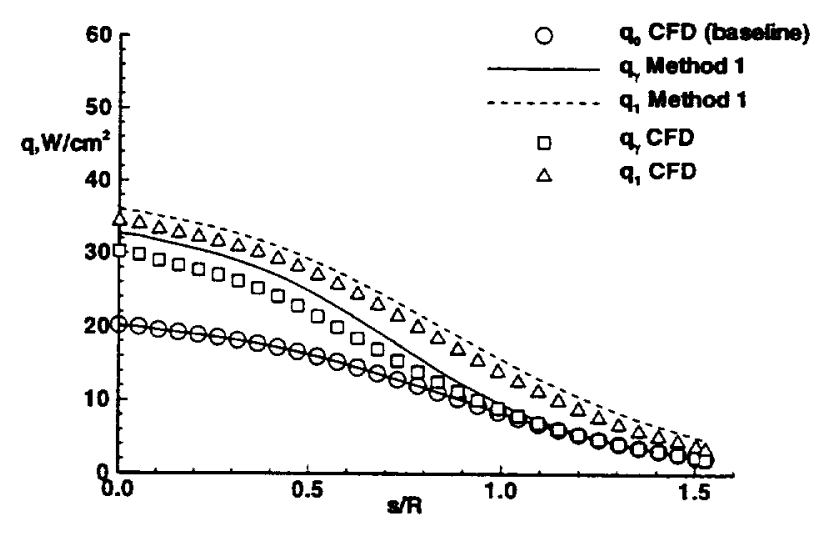

(a) CFD baseline at $\gamma=0$

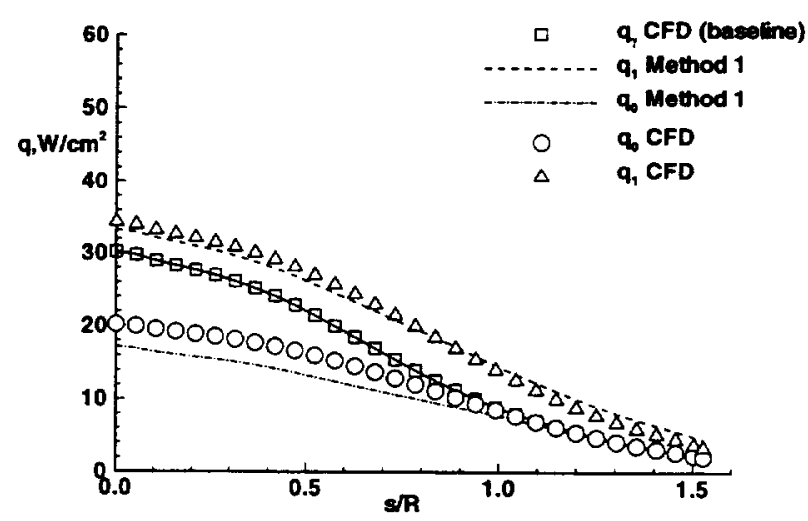

(b) CFD baseline at $\gamma=\gamma(T)$

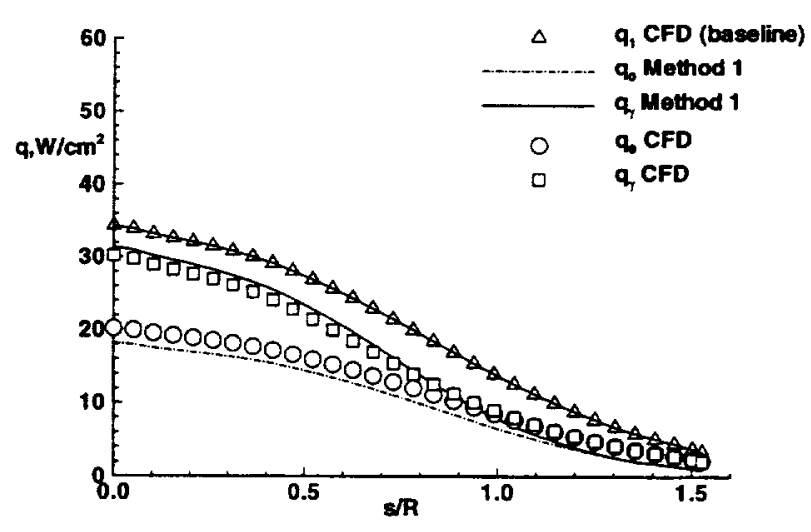

(c) CFD baseline at $\gamma=1$

Figure 2: Comparison of CFD heating levels (symbols) with analytic corrections (lines) derived from CFD baseline for point 2 as function of three surface catalysis models. 


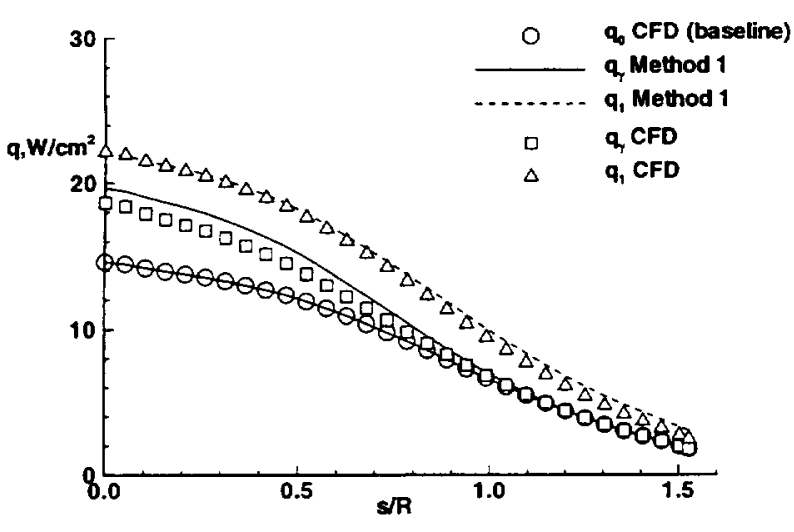

(a) CFD baseline at $\gamma=0$

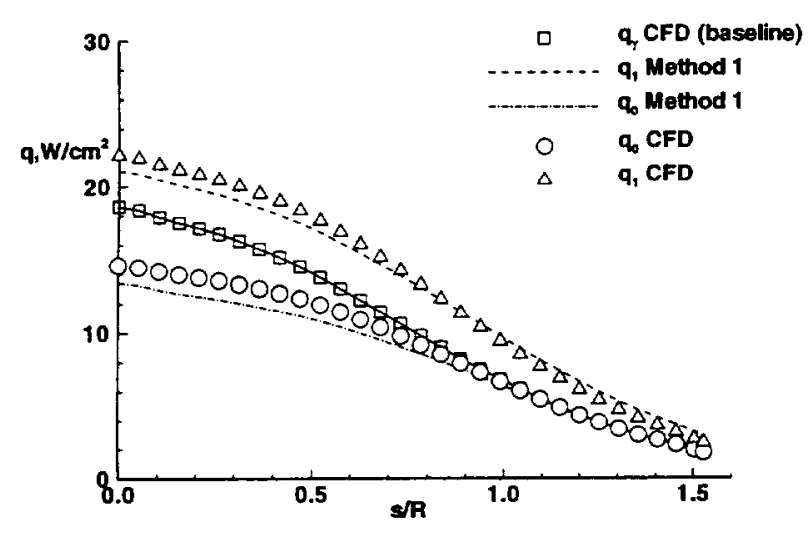

(b) CFD baseline at $\gamma=\gamma(T)$

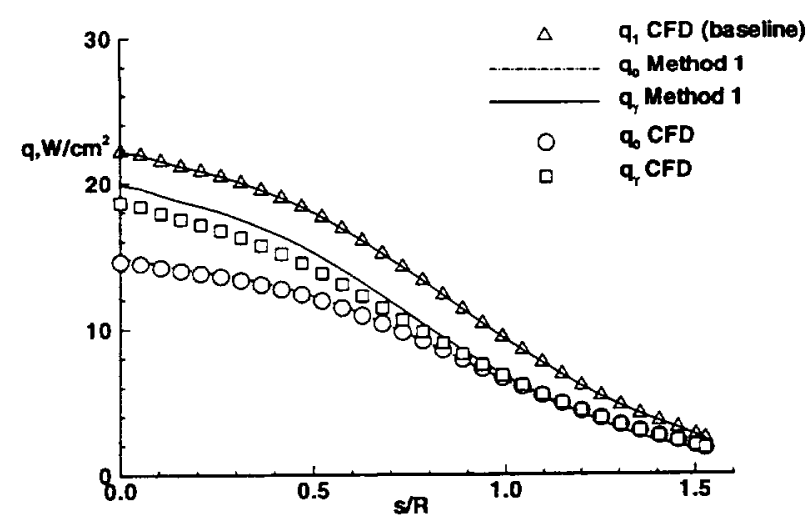

(c) CFD baseline at $\gamma=1$

Figure 3: Comparison of CFD heating levels (symbols) with analytic corrections (lines) derived from CFD baseline for point 3 as function of three surface catalysis models.

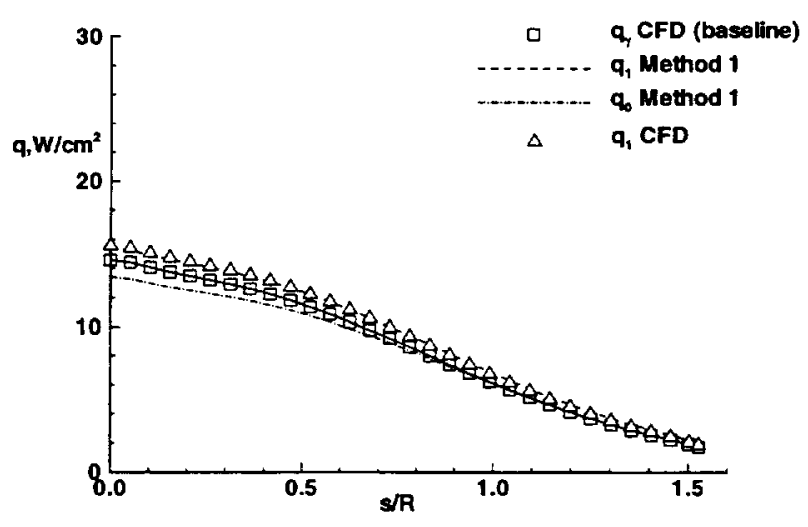

(a) CFD baseline at $\gamma=\gamma(T)$

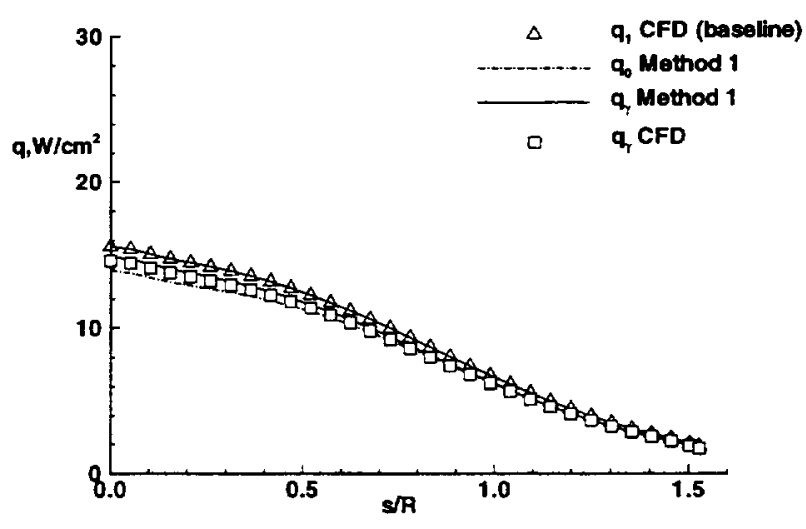

(b) CFD baseline at $\gamma=1$

Figure 4: Comparison of CFD heating levels (symbols) with analytic corrections (lines) derived from CFD baseline for point 4 as function of three surface catalysis models.

Comparisons of Method 1 with CFD at successive trajectory points are presented in Fig. 2 for point 2, Fig. 3 for point 3 , and Fig. 4 for point 4 . A noncatalytic baseline was not run for case 4 because effects of catalysis are so small at this point in the trajectory. In all of these tests, Method 1 corrected heating levels are judged to be acceptable for preliminary design purposes.

\section{Local, Discontinuous Changes to Surface Catalysis}

In Fig. 5, the finite-catalytic solution is treated as the available CFD baseline solution at trajectory point 1. Method 2 is used to post-process the CFD solution to predict the heating levels associated with a local, 
discontinuous change in surface catalysis to a fully catalytic condition at $s / R=\pi / 6$. This result is compared to a corresponding CFD computation with identical wall temperature distribution and grid density. The CFD results in Fig. 5a are computed with 30 cells in 3 degree increments $(\Delta s / R=\pi / 60)$. This grid density would generally be considered adequate to resolve the flowfield over a hemisphere in the streamwise direction. The integral boundary-layer theory (Method 2) shows a spike in predicted heating level of thickness equal to one cell and magnitude more than double the upstream location. (The spike is somewhat rounded because the plotting routine averages cell centered quantities in order to plot at cell corners.) The CFD solution shows only a mild overshoot in heating on this grid. While a discontinuous rise in heating is expected across the interface, the relaxation process back down to the fully catalytic level is not properly resolved. The lack of resolution is not a limitation of the theory; rather, the theory is only applied at CFD grid points in the present algorithm.

A factor of four increase in resolution is introduced in Fig. 5b. Even on this scale, the single point spike of Method 2 indicates that the relaxation process is occurring on a scale still smaller than the current grid resolution $(\Delta s / R=\pi / 240)$. The CFD solution shows a larger overshoot on the finer grid, but is still roughly half the value predicted by Method 2.

An additional, local grid refinement providing a factor 10 more resolution in the vicinity of the interface is applied. The CFD solutions are compared on an expanded scale in Fig. 5c. A relaxation process is finally evident on this scale, though grid convergence has not yet been confirmed. The CFD heating overshoot is approximately $25 \%$ larger on this refined grid, but still approximately $33 \%$ smaller than the Method 2 prediction. Resolution of these differences is still being worked; nevertheless, the application of Method 2 clearly identified a grid resolution problem and serious underprediction of the heating overshoot of the original CFD solution, which rose from 50 to $80 \mathrm{~W} / \mathrm{cm}^{2}$ in the course of two grid refinements.

The present test case provides an extreme example of the overshoot that can be obtained with abrupt changes in surface catalysis. Usually, the juncture would be placed much further from the stagnation point. Theory shows that the relaxation distance increases with running length from the stagnation point, easing somewhat the necessity for local grid refinement. Also, diffusion in a streamwise direction, across the discontinuity, has not been included in the analysis but should serve to reduce the heating spike. Nevertheless, the integral-boundary-layer analysis provides a-priori guidance as to the spacial resolution required to computationally address these issues.

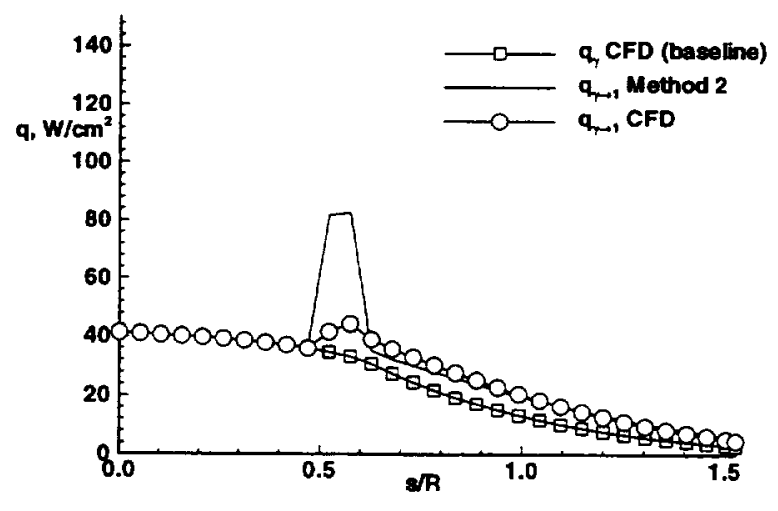

(a) $\Delta s / R=\pi / 60$.

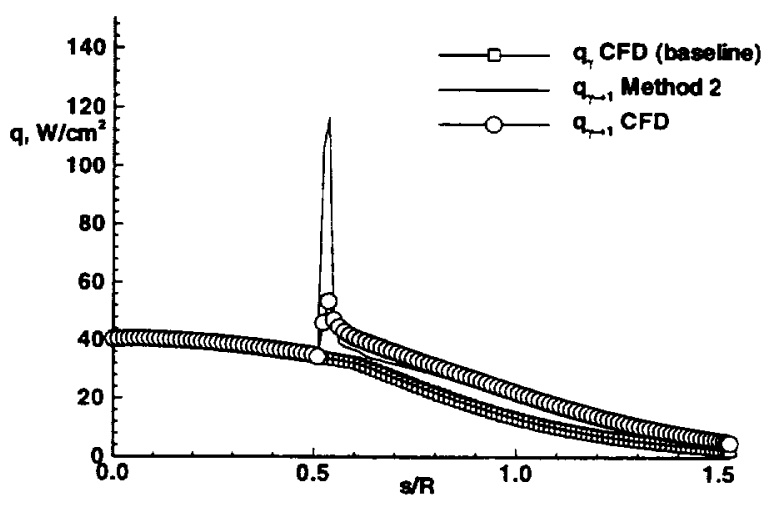

(b) $\Delta s / R=\pi / 240$.

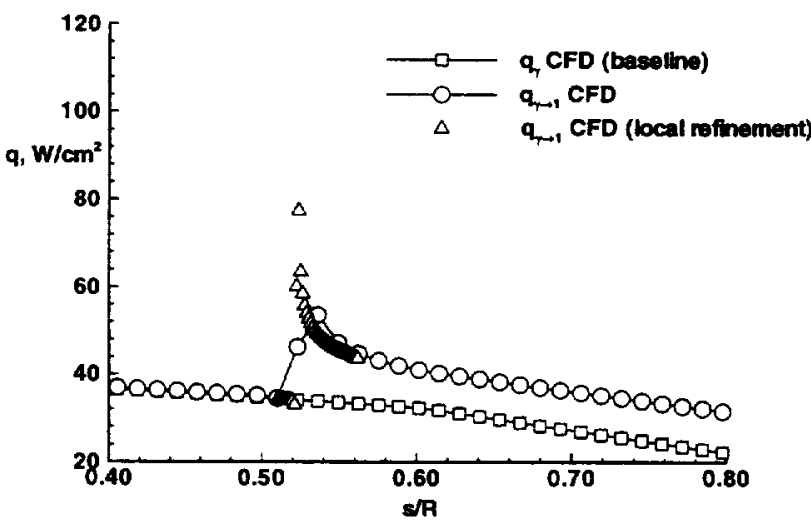

(c) Local refinement with $\Delta s / R=\pi / 2400$.

Figure 5: Comparison of CFD heating levels obtained for an abrupt change in surface catalysis from $\gamma=\gamma(T)$ to $\gamma=1$ with analytic corrections derived from CFD profiles obtained at $\gamma=\gamma(T)$ for point 1 . 


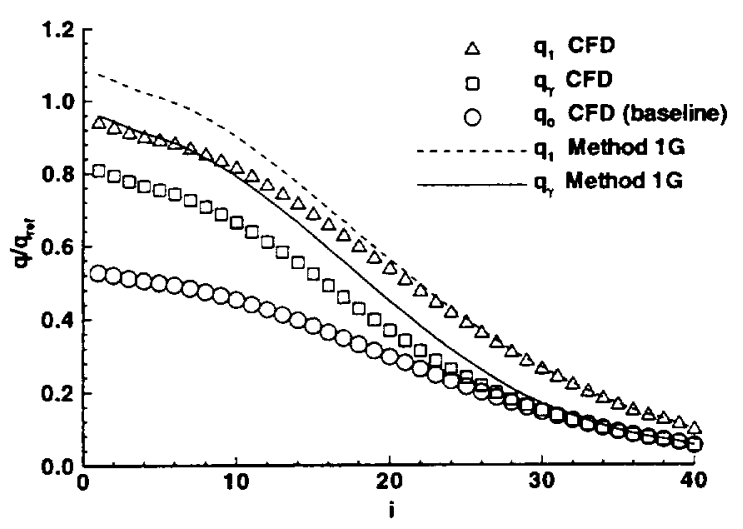

(a) CFD baseline at $\gamma=0$

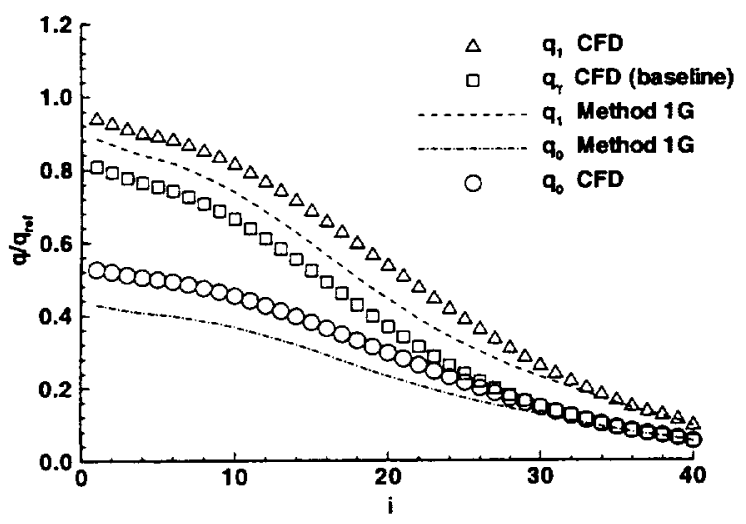

(b) CFD baseline at $\gamma=\gamma(T)$

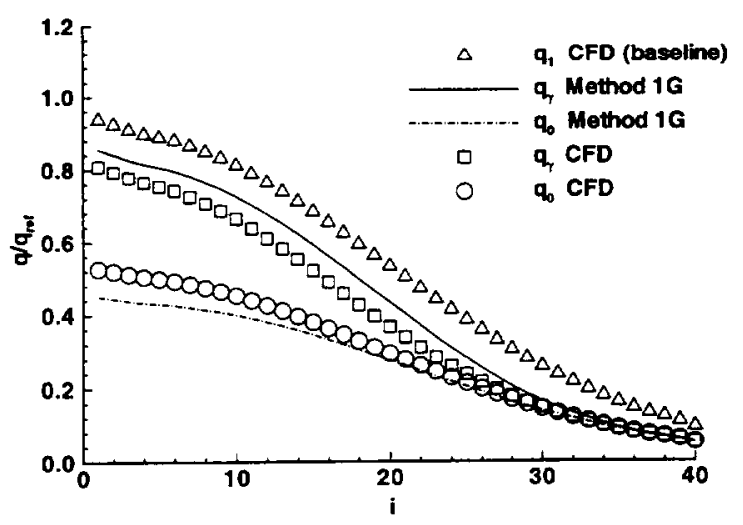

(c) CFD baseline at $\gamma=1$

Figure 6: Comparison of CFD heating levels (symbols) with Method $1 \mathrm{G}$ predictions (lines) for RLV reference sphere at three catalytic surface conditions.
All previous test cases focused on the stagnation region of a sphere where the limiting form of parameter $\chi$ is easily defined. In this section, application of Method 1G to simple and complex configurations is tested. The boundary-layer edge is defined as the point where total enthalpy equals 0.995 of total freestream enthalpy. Velocity at the boundary layer edge is taken as the component that is perpendicular to the surface normal. The Lewis number is calculated with a diffusivity of atomic oxygen diffusing in molecular nitrogen.

In the first case, Method $1 \mathrm{G}$ is applied to analysis of heating to a sphere at Mach 13.5 and $58 \mathrm{~km}$ altitude. Heating rates are plotted as a function of streamwise computational coordinate $i$ in Fig. 6. The CFD cases are fully converged on grids of 128 cells across the shock layer and 40 cells around the body. The CFD solution for each surface catalysis model was computed using the same wall temperature distribution, corresponding to radiative equilibrium with the fully catalytic surface. The integral-boundary-layer corrections to any CFD baseline using Method $1 \mathrm{G}$ are seen to be good predictors of CFD heating levels as a function of surface catalysis away from the stagnation point $(i>20)$ as shown in Fig. 6. However, in the vicinity of the stagnation point the Method $1 \mathrm{G}$ predictions differ from CFD values by as much as $20 \%$ when using a non-catalytic CFD baseline. Predictions are much improved when using a catalytic CFD baseline.

The second test case examines the front and midsections of a Reusable-Launch-Vehicle (RLV) candidate configuration for a flowfield simulation at Mach 25, 45 deg. angle of attack, and $79.6 \mathrm{~km}$. The surface grid representation for the front section is defined with 52 streamise cells and 64 circumferential cells. The surface grid representation for the mid-section is defined with 18 streamwise cells and 77 circumferential cells. The shock layer is resolved with 64 cells. Heating rates for both a fully catalytic wall and a finite-catalytic wall were computed at the respective radiative equilibrium wall temperatures. A secondary iteration was employed in Method $1 \mathrm{G}$ to accomodate the implicit, functional dependence of $\Gamma_{c}$ and $\theta_{w}$ on the wall temperature.

Circumferential heating distributions as a function of computational coordinate $j$ varying from leeside $(j=$ $1)$ to windside $(j=64)$ are presented in Fig. 7 a for the front section. The circumferential cut is from the $i=$ 40 plane which lies far downstream from the nose and upstream of a wing. The windside centerline heating distribution as a function of computational coordinate $i$ varying from the stagnation point $(i=16)$ to the exit plane of the front section $(i=52)$ is presented in Fig. 7b. Method 1G corrections to CFD baseline heating are generally within $5 \%$ of computations. Discepancies in the stagnation region $(i<20)$ are larger but still within $20 \%$ for the fully catalytic case. Here again, 


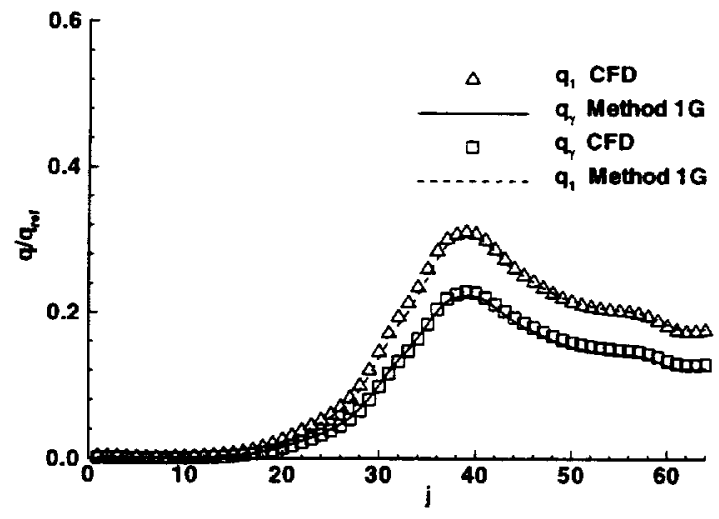

(a) $i=40$ circumferential distribution

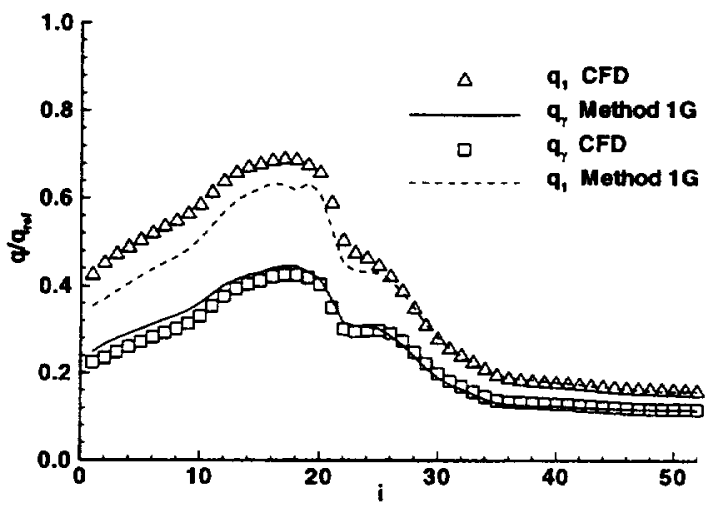

(b) $j=64$ centerline distribution

Figure 7: Comparison of CFD heating levels obtained on front section of RLV with analytic corrections derived from CFD profiles obtained at $\gamma=\gamma(T)$ and $\gamma=1$ at Mach 25 and $79.6 \mathrm{~km}$.

Method 1G corrections are in better agreement with CFD when computed using a fully catalytic-baseline.

The mid-section of the RLV includes the windside surface, the wing, and a small part of the leeside surface. The CFD and integral-boundary-layer results for heating on this section are compared in Fig. 8. Comparisons are generally within a few percent in a circumferential direction around the wing leading edge (Fig. $8 \mathrm{a}$ ), around the windside surface (Fig. $8 \mathrm{~b}$ ) and in a longitudinal direction along the windside centerline (Fig. $8 \mathrm{c}$ ). Comparisons along the wing leading edge show differences that are generally less than $10 \%$.

These results are the first demonstration of the method for a complex flow. Even in the case where significant turning of streamlines occurs for flow ex-

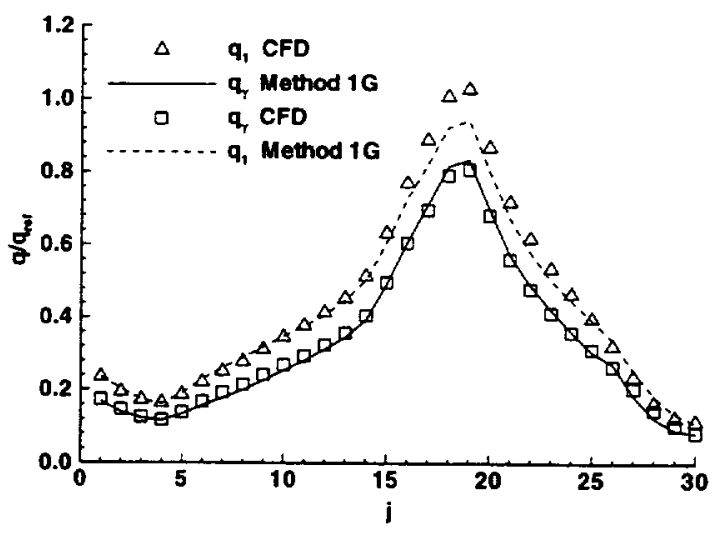

(a) $i=10$ wing

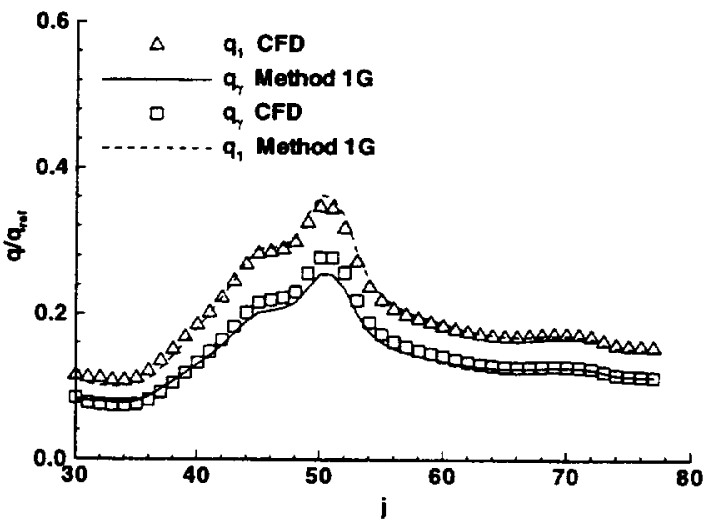

(b) $i=10$ windside

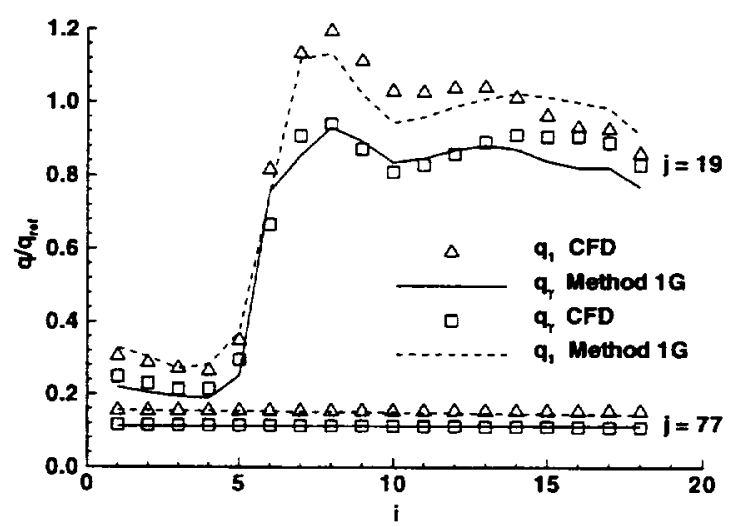

(c) $j=19,77$ centerline and wing leading edge

Figure 8: Comparison of CFD heating levels obtained on mid-section of RLV with analytic corrections derived from CFD profiles obtained at $\gamma=\gamma(T)$ and $\gamma=1$ at Mach 25 and $79.6 \mathrm{~km}$. 
panding around the side of the vehicle $(32<j<48)$ in Fig. $7 \mathrm{a}$ the integral-boundary-layer extrapolation from the baseline CFD computation is an excellent predictor of the CFD result at the off-baseline catalysis model. Though not shown in the figures, the Method $1 G$ predictions for a non-catalytic surface using either the fully-catalytic baseline or the finite-catalytic baseline also agree within a few percent. The largest errors in Method $1 G$ occur in the vicinity of a stagnation point when using a non-catalytic CFD baseline. Some preliminary perturbations of this model suggest that the manner in which nitrogen recombination is handled may be the error source.

The results in Fig. 7 and 8 do not include the correction for dissociation dominated chemistry in the boundary layer as defined in Eq. 23. The correction was evaluated and found to represent less than $1 \%$ of the total heating along the windside centerline for this case. The term grew unreasonably large as the leeside was approached. It appears that the curve fits to tabulated data for the reaction integral ${ }^{2} I_{D}$ are used outside their range of validity. It is expected that this term would be of greater significance if the vehicle were at a smaller angle of attack and more dissociation occurred within the boundary layer.

The differences shown for this test case are thought to be small enough to be acceptable for preliminary thermal protection system design purposes. Some additional tuning of the method is still required in the stagnation region. Also, the consistency of these predictions over a broader range of entry conditions and geometric complexity remains to be established before Method 1G (or a related approach) can be used with confidence in a design mode. The calculations presented here using both Method 1 and Method $1 \mathrm{G}$ offer strong evidence that this design goal is within reach.

\section{Concluding Remarks}

A new approach for combining the insight afforded by integral boundary-layer analysis with comprehensive (but time intensive) computational fluid dynamic (CFD) flowfield solutions of the thin-layer Navier-Stokes equations is presented. The approach extracts CFD derived quantities at the wall and at the boundary layer edge for inclusion in a post-processing boundary-layer analysis, valid for most of the acreage on a realistic hypersonic vehicle. It allows a designer at a workstation to address two questions, given a single, baseline CFD solution: (1) How much does the heating change for a thermal protection system with different catalytic properties than was used in the baseline CFD solution? (2) How does the heating change at the interface of two different TPS materials with an abrupt change in catalytic efficiency?

Global changes in surface catalysis lead to global changes in heating rates. Two methods were developed in order to quantify these changes from a baseline, CFD solution. Method 1 is restricted to the stagnation region of vehicles. Method $1 \mathrm{G}$ is a straightforward extension of Method 1 which is applicable to the entire vehicle. Rather than employing limiting forms of the boundary-layer equations or integrating along streamlines, it solves for an integral-boundary-layer streamline parameter to match the local CFD baseline heating rate. This parameter is then used to predict heating rates at off-baseline values of surface catalysis. The influence of boundary-layer chemistry can be included in these analyses, but was found to be small in the test cases treated herein. Off-baseline predictions of heating rates by both Method 1 and $1 \mathrm{G}$ were generally in very good agreement with CFD solutions, with Method $1 \mathrm{G}$ appearing somewhat superior in the limited tests performed to date. The differences shown for this test case are thought to be small enough to be acceptable for preliminary thermal protection system design purposes. Some additional tuning of the method is still required in the stagnation region. Also, the consistency of these predictions over a broader range of entry conditions and geometric complexity remains to be established before Method $1 G$ (or a related approach) can be used with confidence in a design mode. The calculations presented here using both Method 1 and Method $1 \mathrm{G}$ offer strong evidence that this design goal is within reach.

Abrupt, discontinuous changes in surface catalysis lead to large, local changes in heating rates that must relax to the new level dictated by the catalytic efficiency of the new surface. Method 2, applicable only in the stagnation region, predicted large heating spikes associated with an abrupt change in surface catalysis. The spikes were larger than initially predicted by CFD. However, the relaxation zone, as defined by the integral-boundary-layer method, was not adequately resolved and grid refinement tended to bring the CFD result in better agreement with that predicted by Method 2. Remaining differences between Method 2 and CFD are likely due to numerical diffusion across the discontinuity and require further study. An extension of Method 2 to Method 2G, applicable outside the stagnation region, should be straightforward but has not yet been tested. 


\section{$\underline{\text { References }}$}

${ }^{1}$ Inger, G. R., and Gnoffo, P. A., "Hypersonic Entry Heating with Discontinuous Surface Catalycity: A combined Analytic/CFD Approach," AIAA Paper 962150, June 1996.

${ }^{2}$ Inger, G. R., "Nonequilibrium Boundary Layer Effects on the Aerodynamic Heating of Hypersonic Waverider Vehicles," Journal of Thermophysics and Heat Transfer, Vol. 9, No. 4, October-December 1995, pp. 595-604.

${ }^{3}$ Inger, G. R., "Nonequilibrium Stagnation Point Boundary Layers with Arbitrary Surface Catalycity," AIAA Journal, Vol. 1, No. 8, August 1963, pp. 17761784.

${ }^{4}$ Inger, G. R., and Elder, J., "Recombination Dominated Nonequilibrium Heat Transfer to Arbitrarily Catalytic Hypersonic Vehicles," Journal of Thermophysics and Heat Transfer, Vol. 5, No. 4, 1992, pp. 449455.

${ }^{5}$ Gnoffo, P. A., "Point-Implicit Relaxation Strategies for Viscous, Hypersonic Flows," in Computational Methods in Hypersonic Aerodynamics (Murthy, T. K. S., ed.), Computational Mechanics Publications, pp. 115-151, Kluwer Academic Publishers, 1991.

${ }^{6}$ Gnoffo, P. A., "Upwind-Biased, Point-Implicit Relaxation Strategies for Viscous, Hypersonic Flows," AIAA Paper 89-1972, 1989.

${ }^{7}$ Gnoffo, P. A., "An Upwind-Biased, PointImplicit Relaxation Algorithm for Viscous, Compressible Perfect-Gas Flows," NASA TP 2953, February 1990.

${ }^{8}$ Gnoffo, P. A., "Code Calibration Program in Support of the Aeroassist Flight Experiment," Journal of Spacecraft and Rockets, Vol. 27, No. 2, March-April 1990, pp. 131-142.

${ }^{9}$ Weilmuenster, K. J., and Gnoffo, P. A., "Aeroassisted Flight Experiment Aerodynamic Characteristics at Flight Conditions," Journal of Spacecraft and Rockets, Vol. 27, No. 6, November-December 1990, pp. 684686.

${ }^{10}$ Thompson, R. A., and Gnoffo, P. A., "Application of the LAURA Code for Slender Vehicle Aerothermodynamics," Journal of Spacecraft and Rockets, Vol. 29, No. 1, January-February 1992, pp. 16-23.

${ }^{11}$ Weilmuenster, K. J., Gnoffo, P. A., and Greene, F. A., "Navier-Stokes Simulations of Orbiter Aerodynamic Characteristics Including Pitch Trim and Bodyflap," Journal of Spacecraft and Rockets, Vol. 31, No. 3, May-June 1994, pp. 355-366.

${ }^{12}$ Gnoffo, P. A., Weilmuenster, K. J., and Alter, S. J., "Multiblock Analysis for Shuttle Orbiter Reentry Heating from Mach 24 to Mach 12," Journal of Spacecraft and Rockets, Vol. 31, No. 3, May-June 1994, pp. 367-377.
${ }^{13}$ Roe, P. L., "Approximate Riemann Solvers, Parameter Vectors, and Difference Schemes," Journal of Computational Physics, Vol. 43, October 1981, pp. 357-372.

${ }^{14}$ Harten, A., "High Resolution Schemes for Hyperbolic Conservation Laws," Journal of Computational Physics, Vol. 49, No. 2, February 1983, pp. 357-393.

${ }^{15}$ Yee, H. C., "On Symmetric and Upwind TVD Schemes," NASA TM 88325, 1986.

${ }^{16}$ Stewart, D. A., Leiser, D. B., Kolodziej, P., and Smith, M., "Thermal Response of Integral Multicomponent Composite Thermal Protection Systems," AIAA Paper 85-1056, June 1985.

${ }^{17}$ Goulard, R. J., "On Catalytic Recombination Rates in Hypersonic Stagnation Heat Transfer," Jet Propulsion, Vol. 28, No. 11, 1958, pp. 737-745. 


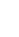


